\title{
Correction to: Superalloys 2020
}

Sammy Tin, Mark Hardy, Justin Clews, Jonathan Cormier, Qiang Feng, John Marcin, Chris O'Brien, and Akane Suzuki

\section{Correction to:}

S. Tin et al. (eds.), Superalloys 2020, The Minerals, Metals \& Materials Series, https://doi.org/10.1007/978-3-030-51834-9

The original version of the following chapters was inadvertently published with error.

In chapter 6, equation 6, the incorrect word "quad" has been removed.

In chapter 32, the updated Abstract has been included.

The Chapters and book have been updated with the above changes. 